\title{
Synthetic Fabrication of Biomimetic Artificial Skin Tissue
}

\author{
Joo han Kim', Jooyoub Lee ${ }^{2}$ and Suw Young Ly ${ }^{3 *}$ \\ ${ }^{1}$ Department of Chemical Engineering, DaeJin University, Pocheon \\ ${ }^{2}$ Deparment of Fire and Disaster Prevention Engineering Jungwon University \\ ${ }^{3}$ Biosensor Research Institute, Seoul National University of Science and Technology Seoul \\ 232 Korea \\ *Corresponding author
}

\section{A B S T R A C T}

Human skin cells have limited mechanical and biochemical resistance properties. As such, it can be difficult for them to exist under extreme

Keywords

Synthetics,

Fabrication,

Biomimetics,

Assay, Artificial

skin tissue

Article Info

Accepted:

15 July 2019

Available Online:

10 August 2019 conditions due to a lack of resistance resulting from factors including chemical stabilities, abrasion strength, impact stress and other biological activities. In order to combat these problems, we have synthesized a highly specialized physicochemical skin analogue polymerization. This sees functional groups prepared on an electrochemical magnetic field and then synthesized in human-like water dispersion electrolyte conditions. The mole ratio was changed so as to enhance the high impact resistance, oxidation resistance and membrane stability. The results of the physical properties of mechanical, chemical and biological resistance were then compared with human cell strength. The results of the study confirmed the resistance of the skin film coating in real-time in vitro skin of the human face, and can therefore be used in extreme conditions such as an in-vitro physico-resistance.

\section{Introduction}

In vivo human skin is vulnerable to mechanical and biological skeletal maintenance issues that can arise under a variety of different extreme conditions. Among these, physical and biochemical shock resistance is limited (1) and is unable to escape from normal temperature and normal pressure conditions (2). In order to counter these problems, we have synthesized strong physicochemical resistant skins like polymers that are able to successfully maintain in vitro skeletal situations even under extreme conditions. Here, synthetic electrolytes were also performed through oil dispersion and a human friendly water dispersion electrolyte was used. Functional confirmation was achieved in terms of physical tensile, impacted strength, viscosity, infrared absorption, and 
line of sight scattering. Under these conditions, the synthesized membrane was then applied to the skin surface coated sensors, and here similar skin functions were confirmed by the redox potential assay. Moreover, the in vivo diagnostic instrumental circuit was used for the electrochemical amplifier systems $(3,4)$ by our circuits. The functional assay was performed by in vivo (5) or in vitro coated sensors $(6,7)$. Furthermore, modification amplifiers $(8,9)$ can be used for human-like modified carbon compounds (10), nanotubes $(11,12)$, and more. The in vivo vital current was obtained through chronocyclic techniques, stripping (13), micro sensing (14) and immobilization (15) for signal amplification. Moreover, synthesized film immobilizations were applied directly to the skin (16), working electrodes (17), counters (18) and reference three electrodes (19, 20). Similar skin functions responded instantaneously to biochemical conditions such as neurotransmission, external pressure, shock, and temperature response under optimal conditions. In addition, these results can be applied to artificial skin, skin barriers, medical skin substitutes, and body sensors.

\section{Materials and Methods}

\section{Synthetic method}

\section{Molecular structure of standards}

The reagents of standards were prepared as follows. A Sigma Aldrich purity of $99.0 \%$ was used. Moreover, the synthesis reaction vessel was made from $100 \% \mathrm{SiO}_{2}$ quartz crystals fabricated in a laboratory so as to remove any possible interference effects. All syntheses were performed at a standard room temperature of $25{ }^{\circ} \mathrm{C}$. Water was then added after the first purification of tap water. This, in turn, was purified by repeatedly distilling 18 $\mathrm{M}$ OHM resistance. The metal container was blocked from the possible problem of the elution of impurities by using $100 \%$ stainless steel. All of the reagents that can be seen in (a) $\sim$ (g) used $95.0 \%$ or higher purity of Aldrich Sigma Co. Nevertheless, if necessary, further purification was also performed so as to remove any interfering effects. The molecular weight of $(b) \sim(d)$ ranged from 58.6 to 1513 $\mathrm{g} / \mathrm{mol}$ while the density ranged between $0.72 \sim$ $1.05 \mathrm{~g} / \mathrm{Cm}^{3}$ at $20^{\circ} \mathrm{C}$. Both solubility and vaper pressure were possible for water dispersion and oil dispersion. In addition, it was also possible to synthesize these at both the melting point and boiling point of the isotherm. For these reasons, no other additional devices were used (Fig. 1).

\section{Experimental synthetic fabrication}

The basic oil dispersion polymer mixing ratio was investigated through the trials of a variety of different methods. The statistically confirmed optimum molar ratios are shown in the figure 2. It was stabilized in the range of $80 \sim 85^{\circ} \mathrm{C}$ to prevent high temperature evaporation. The reaction time was activated within 8 hours and further optimal sequential substitutions are shown in figure 2 .

The synthesis molar ratio and the optimal temperature were determined through a close analysis and with reference to the published common literature. However, the results optimized for physical functionality are the same a, s presented in table (a) (Fig. 2). Following this, DMPA was then added at $80 \sim$ $85^{\circ} \mathrm{C}$ for 6 hours to allow for sufficient synthesis time.

In addition, TRA and water were added so as to allow stabilization at $40^{\circ} \mathrm{C}$ in the same manner. TEA, EDA, and defoamer, were sequentially converted in order to enhance ductility, abrasion, resistance and physicochemical bonding strength. The changed mole ratios are recorded and presented in (b) and (c). Moreover, the results of the bio, physical and chemical properties and their level of impact strength was subsequently obtained. 


\section{Results and Discussion}

\section{Diagnostic potential assay by using cyclic redox scanning}

Undersynthetic electrolytic conditions, a cyclic redox scanning was performed to examine the sensors' response to the three electrodes working, counter and reference probes. Moreover, the conductive strength of the electrolyte activity was also analyzed.

Under the aforementioned synthetic electrolytic paste conditions, a redox scanning was performed on the three electrode systems. All of the figures, (a) (d), produced normal wave sigmoid current responses. The potential windows of $-2.0 \mathrm{~V}$ initial, and $2.0 \mathrm{~V}$ switching potentials were also performed at this time. Moreover, a cyclic redox can be performed under synthetic paste conditions. In this regard figure (a) demonstrates a simple sigmoid redox scanning - a water dispersion paste appeared with a very fast redox strength. Following on from this (b) contains the other samples of $-1.4 \mathrm{~V}$, and $-0.4 \mathrm{~V}$ oxidation peak potentials that were obtained. However, the reduction potentials not obtained and the current ranges are $0.2 \times 10^{-3} \mathrm{~A} \sim 0.35 \times 10^{-3} \mathrm{~A}$. Furthermore the other scanning of (c) and (d) is the same as the repeated normal wave that appeared, (c) = figure 2-1(b) and (d) = figure 2-1(c), in which the sequential synthetics can be seen to produce oxidation activity but not any noticeable reduction in strength. The physical properties of other conditions were also investigated and analyzed at this time.

\section{Adhesive strength measurement}

The physical tensile strength of the composite synthetic membrane was measured after it had been attached to the surface of the unit metal. After this, the molecular bond strength was cut. The adhesive used for the membrane was
Resinlab N109w13300 Ellsworth Dr Germantown, WI 53022. All measurements were performed at a standard room temperature of $20^{\circ} \mathrm{C}$. The adhesive force measuring devices used in this experiment were the Defelsko Posi Test ATM50C / ASTM C1583 / Pull-off Adhesion Testers. Moreover, in every situation the Posi Test Adhesion Tester pressure system was calibrated and certified to $\pm 1 \%$ accuracy using an NIST traceable load cell Self-aligning aluminum dolly. This was used for it enables highly accurate measurements on smooth or uneven surfaces. Because the equipment ishigrade, the experimental pressure sensors ensure continued accuracy. The Pull Rate Indicator allows the operator to monitor and adjust the rate of pull in accordance with standardized international test methods. Furthermore, the easily selected dolly sizes, changesin measurement units or store readings, can be adjusted at the mere touch of a button. Conversion charts were not required because the tester automatically calculates the pressure based on the dolly size. The inexpensive nature of the equipment means that single-use dollies eliminate cleaning for re-use and can be kept as part of a permanent record. Finally, each kit comes with everything needed to successfully carry out the testing process. The results of the experiments are shown in the graphs below,

The attachment strength was represented in photographs (A), (B), and (C) for 9th repeated iterations. Figure (A) presents the median values of (201 psi, $4.2 \mathrm{sec}, 1000 \mathrm{psi}, 24.3 \mathrm{sec}$, $1000 \mathrm{psi}$ and $23.0 \mathrm{sec})$ while sample 2 recorded those that were repeated (B): (737 psi $9.3 \mathrm{sec}, 73.9 \mathrm{psi}, 10.6 \mathrm{sec}, 647 \mathrm{psi}$, and 9.6 sec). Finally, (C) shows the other samples of (649 psi, $9.6 \mathrm{sec}$ ) strength respectively. During this process the duration was repeated each time to try and ensure accuracy. 
Fig.1 Molecular structure of the standard functional group, (a): 2,2-Bis (hydroxymethpropionic acid, (b): Polypropylene glycol, (c): Isophorone.(d): Dibutyltin dilaurate, (e): Acetone, (f):

Triethylamine, (g): Ethylenediamine

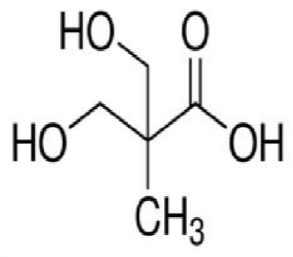<smiles>CC(CO)CO</smiles>

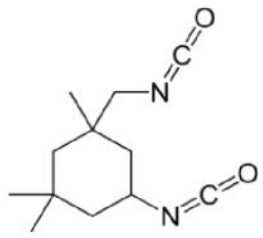

(a)

(b)

(c)

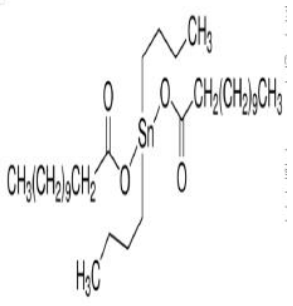<smiles>CC(C)=O</smiles>

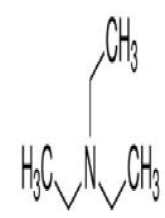

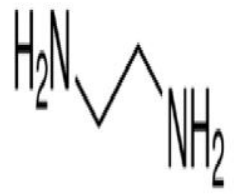

(d)

(e)

(f)

(g)

Fig.2 Sequential change of (a), (b) and (c) molar ratio of water dispersion synthesis table
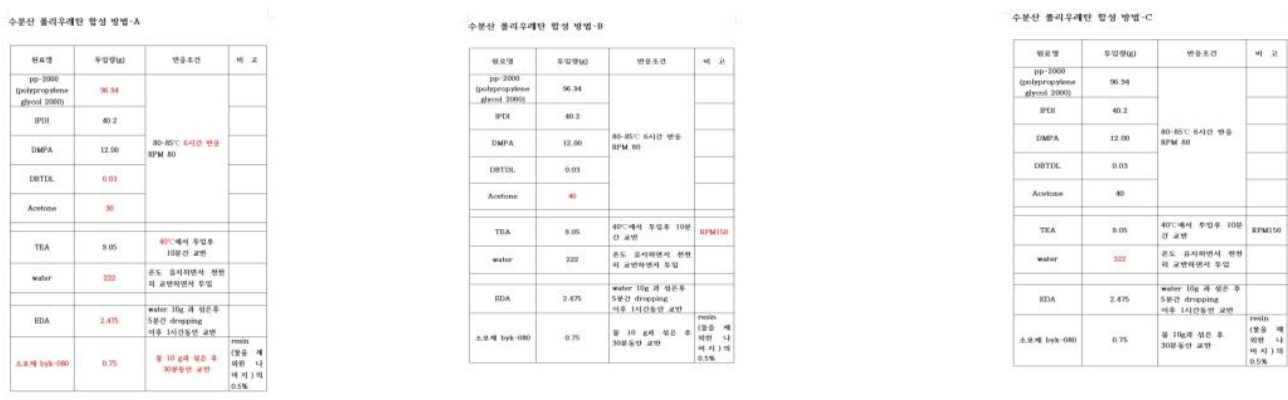

(a)

(b)

(c) 
Fig.3.1 Cyclic voltammetric normal redox activity for water dispersion synthesis sequential steps of (a), (b), (c), (d on graphite three electrode systems, using bio-electrochemical instruments-2, our circuit, nut not any electrolytes
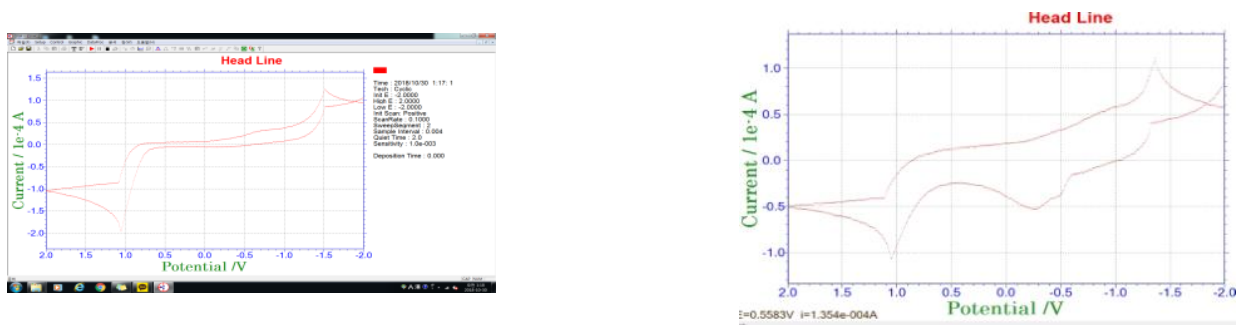

(a)

(b)
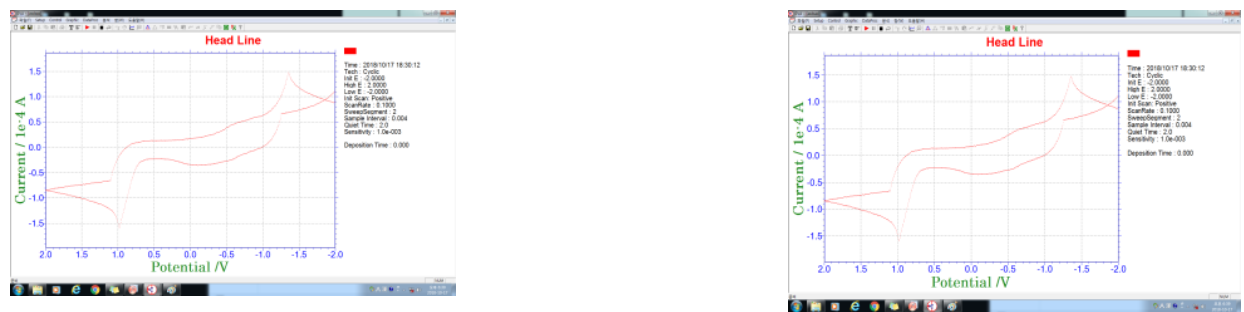

(c)

(d)

Fig.3.2 (A): The median value of the repeated 3 times for sample one, (B): The median value of sample 2 using the same method. (C) The median value of sample 3 using the same method as

(A) and (B)
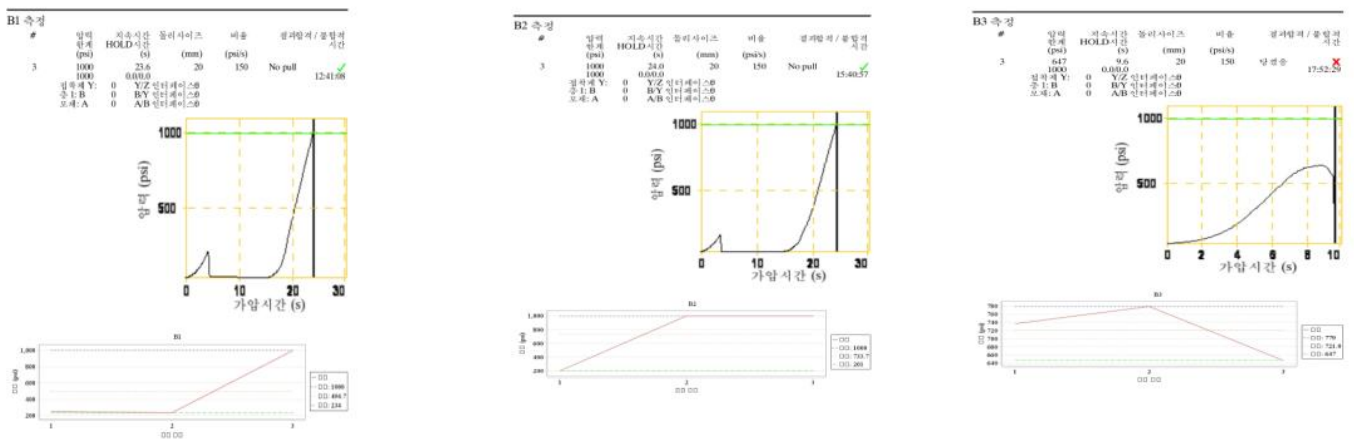
(A)
(B)
(C)

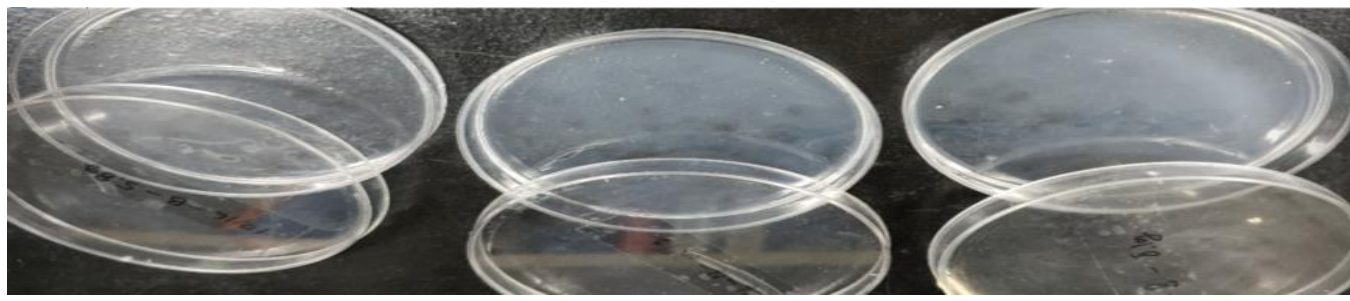


In vivo diagnostic muscle sensing applications

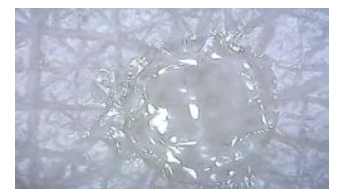

(a)

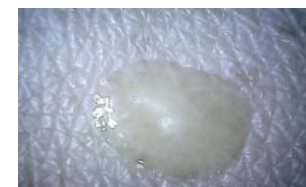

(b)

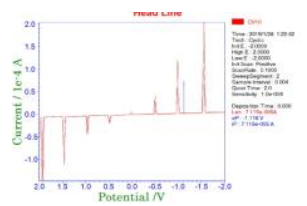

(c)

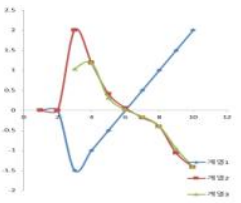

(d)

Figure 3.3. in vitro muscle coating artificial skin sensor, 20x magnification enlarged photo, skin of a 25 year-old male (a): on the right hand of $5 \mathrm{~mm}$ diameter $0.5 \mathrm{~mm}$ thick film, (b): Photograph on the forehead skin muscle sensor under the same conditions as (a). (c): contraction of back muscles relaxation redox neuron current, $0.5 \mathrm{~V}$ sequential repeat. $(\mathrm{d})$ : peak height measurement of graph (c).

Nevertheless, all of these results are applicable to the physical strength of artificial skin films. Therefore, we experimented directly on the surface of human skin. The picture below is of a biomimetic virtual tissue of (a), (b), and (c). It is designed to show the transparent spectrums through the line of sight. These can then be tested for muscle sensing applications for in vivo neuro diagnostics.

\section{In vivo diagnostic muscle sensing applications}

The nerve current responses for potential scanning was obtained through the use of virtual skin coated sensors that were applied and can be seen in figure 3.3 (a). The first is a photograph of a young man with a synthetic coating on his right hand. The second, (b), is a coated picture that has been applied to the skin on his forehead. During this procedure, a 3-electrode system to examine the physicochemical nerve responses using our circuits, as well as bio-electrochemical analyzer- 2 instruments, were used. The potential scanning was $2.0 \mathrm{~V}$ to $-2.0 \mathrm{~V}$, and the circulating reversible potential was used at this time. Moreover, the scanning speed was fixed at a $50 \mathrm{mv} / \mathrm{sec}$ as this was carried out. The nerve currents relaxed the muscles in $0.5 \mathrm{~V}$ increments. At this time, the potential difference diagram showed a definite redox intensity as shown in (C). The current range appeared to present evidence of a redox symmetry in the results with 9 points of the oxidations peak current and 9 points of the reduction peak current obtained as shown in Figure (D) at $1.0 \times 10^{-4} \mathrm{~A}$ to $1.0 . \times 10^{-7} \mathrm{~A}$. Here, the results can be seen to demonstrate a response to muscle neuro-diagnostics of the skin on the surface of the hand (a) and forehead (b). Moreover, the in vivo computer brain interfaced scanning which was performed on other muscles on various other parts of the body's skin and the same results were obtained; however, they are not shown here,

The mechanical ductility, elasticity and acid base resistance of the synthesized artificial skin were all notably higher than that of human cells. Moreover, the redox potentials reacted in a reversible manner. Nevertheless, in some results it was not possible to see the reduction potential. Therefore, it demonstrated electrical characteristics similar, in some way at least, to human skin. There were no significant interference effects on the human skin application due to the water dispersion synthesis. Furthermore, the strength of the intermolecular bonding force was stronger in the adhesion test. The visible transmittance of the synthetic film is not less 
than $80 \%$ and does not block the wavelengths of $340 \mathrm{~nm}$ to $750 \mathrm{~nm}$, other infrared absorption, viscous tensile forces, and other elements. The false eyelashes may be used as a virtual skin protection film or skin replacement film.

\section{References}

1. Siti Noor Azizzati Mohd Noor, Jamaluddin Mahmud. A Review on Synthetic Skin: Materials Investigation, Experimentation and Simulation, Advanced Materials Research Vols. 915-916, 2014, 858-866.

2. Toribio F. Otero, Jose G. Martinez, Physical and chemical awareness from sensing polymeric artificial muscles. Experiments and modeling, Progress in Polymer Science, Volume 44, May 2015, 62-78.

3. Suw Young Ly, Young Sam Jung, Chang Hyun Lee, Bang Won Lee, Administering Pesticide Assays in In Vivo Implanted Biosensors, Aust. J. Chem. 2008, 61, 826-832.

4. Suw Young Ly, Diagnosis of copper ions in vascular tracts using a fluorine-doped carbon nanotube sensor,Talanta 74 (2008) 1635-1641.

5. Suw Y. Ly, Diagnosis of Diabetes in a Diabetic Patient's Urine and Blood Using a Combination Electrode with a Ubiquitous Handheld Analyzer, Current Analytical Chemistry, 2009, 5, 59-64

6. Suw Young Ly, So Dam Yoo, Seung Kyu Chun, Detection of Helicobacter pylori DNA in preliminary stage gastric cancer cells, Pathology, 2012, 44, 251-254

7. Suw Young Ly, Voltammetric analysis of DL- $a$-tocopherol with a paste electrode, J Sci Food Agric, 2008, 88, 1272-276

8. Suw Young Ly, Young Sam Jung, Sung Kuk Kim, Hyun Kuy Lee, Trace Analysis of Lead and Copper Ions in
Fish Tissue Using Paste Electrodes, Analytical Letters, 40: 2683-692, 2007

9. Suw Young Ly, Sang Su Song, Sung kuk Kim,Young Sam Jung, Chang Hyun Lee, Determination of Ge(IV) in rice in a mercury-coated glassy carbon electrode in the presence of catechol,Food Chemistry 95, 2006, 337-343

10. Suw Young Ly, Jung Eun Kim, Woo Yeon Moon,Diagnostic assay of carcinoembryonic antigen tumor markers using a fluorine immobilized biosensor with handmade voltammetric circuit, European Journal of Cancer Prevention 2011, 20, 58-62

11. Suw Young Ly, Hai Soo Yoo, Joon Young Ahn, kwang hee Nam, Picomolar assay of riboflavin in human urine using voltammetry, Food Chemistry 127,270-274,2011.

12. Suw Young Ly, Hai Soo Yoo, Seung Kyu Chun, Detection of trace metal in distilled alcoholic drinks, Food Chemistry 137, 168-171, 2013.

13. Suw Young Ly, Myoung Ho Shin, Chang Hyun Lee, Jin Hui Lee, Mi Sook Kim, Sang Woo Ji and Dong won Park, Detection of trace cobalt ions in in vivo plant cells using a voltammetric interlocking system, Water Science \& Technology 67.6, 1339-1344,2013.

14. Suw Young Ly, Dal woong Choi, Implementation of a Bio circuit Implants for Neuro transmitter Release During Neuro Stimulation, Current Neuro vascular Research,10, 238-246, 2013.

15. Suw Young Ly, Dal Woong Choi, Dae Hong Kim, Diagnostic Assay of Glucose in Diabetes Patients' Urine, Sensor Letters 11, 1996-1999,2013.

16. Suw Young Ly, Detection of dopamine in the pharmacy with a carbon nano tube paste electrode using voltammetry, Bioelectro chemistry 68, 232-236.2005. 
17. Ly SY, Jung YS, Kim MH, Kwon HanI, Jung WW, et al. Determination of Caffeine Using a Simple Graphite Pencil Electrode with Square Wave Anodic Stripping Voltammetry. Microchim Acta, 146, 207-213, 2004.

18. Ly SY, Kim DH, Kim MH, Square wave cathodic stripping voltammetric analysis of RDX using mercury film plated glassy carbon electrode. Talanta 58: 919-926, 2002.
19. Suw Young Ly, Min Joon Kim, Diagnostic Assay of Chromium (VI) in the Ex Vivo Fluid of the Urine of a Smoker Using a Fluorine-Doped Hand made Sensor, Journal of Clinical Laboratory Analysis 23 : 82-87, 2009.

20. Suw Young Ly, Jin-Hui Lee, Dong Ho Jung, Radioactive uranium measurement in vivo using a handheld interfaced analyzer, Environmental Toxicology and Chemistry, 29, 10251030.

\section{How to cite this article:}

Joo han Kim, Jooyoub Lee and Suw Young Ly. 2019. Synthetic Fabrication of Biomimetic Artificial Skin Tissue. Int.J.Curr.Microbiol.App.Sci. 8(08): 2144-2151.

doi: https://doi.org/10.20546/ijcmas.2019.808.250 